\title{
A 100-Element HBT Grid Amplifier
}

\author{
Moonil Kim, Member, IEEE, Emilio A. Sovero, Jonathan B. Hacker, Student Member, IEEE, \\ Michael P. De Lisio, Member, IEEE, Jung-Chih Chiao, Shi-Jie Li, David R. Gagnon, \\ Member, IEEE, James J. Rosenberg, and David B. Rutledge, Fellow, IEEE
}

\begin{abstract}
A 100-element 10-GHz grid amplifier has been developed. The active devices in the grid are chips with heterojunction-bipolar-transistor (HBT) differential-pairs. The metal grid pattern was empirically designed to provide effective coupling between the HBT's and free space. Two independent measurements, one with focusing lenses, the other without, were used to characterize the grid. In each case, the peak gain was $10 \mathrm{~dB}$ at $10 \mathrm{GHz}$ with a 3-dB bandwidth of $1 \mathrm{GHz}$. The input and output return loss were better than $15 \mathrm{~dB}$ at $10 \mathrm{GHz}$. The maximum output power was $450 \mathrm{~mW}$, and the minimum noise figure was $7 \mathrm{~dB}$. By varying the bias, a signal could be amplitude modulated with a modulation index as large as 0.65 . Tests show that the grid was quite tolerant of failures-the output power dropped by only $1 \mathrm{~dB}$ when $10 \%$ of the inputs were detuned. The grid amplifier is a multi-mode device that amplifies beams of different shapes and angles. Beams with incidence angles up to $30^{\circ}$ were amplified with less than a 3-dB drop in gain.
\end{abstract}

\section{INTRODUCTION}

Q UASI-OPTICAL GRIDS offer the possibility of largescale power combining of solid-state devices without the loss limitations of transmission-line combiners. A variety of grids have been demonstrated, including detectors [1], phase shifters [2], [3], frequency multipliers [4], [5], oscillators [6], [7], amplifiers [8], [9], mixers [10], and switches [11], [12]. The first grid amplifier [8] was a device with 25 pairs of MESFET's that amplifies a beam, with cross-polarized input and output (Fig. 1). This configuration helps to prevent the grid from oscillating, and allows polarizers to tune the input and the output independently. The transistors were wired as differential pairs, with the two sources tied together. The two gates were the inputs, and the two drains were the outputs. The grid showed a gain of $11 \mathrm{~dB}$ at $3.3 \mathrm{GHz}$. It had metal strips on the back for biasing, and resistors and pins to connect to packaged transistors on the front. The strips were

Manuscript received November 1, 1992; revised February 1, 1992. The work at Caltech was supported by the Army Research Office, the Northrop Corporation, the Naval Weapons Center in China Lake, CA, and the Rockwell International Trust, and the Jet Propulsion Laboratory.

M. Kim was with the Department of Electrical Engineering, California Institute of Technology, Pasadena, CA 91125 . He is now with the Central Research Laboratory, Gold Star, 16 Woomyen-Dong, Seocho-Gu, Seoul $137-40$, Korea.

E. A. Sovero is with the Science Center, Rockwell International Corporation, 1049 Camino Dos Rios, Thousand Oaks, CA 91385.

D. R. Gagnon is with the Naval Air Warfare Weapons Division, Naval Weapons Center, China Lake, CA 93555-6001.

J. J. Rosenberg is with the Engineering Department, Harvey Mudd College, Claremont, CA 91711.

J. B. Hacker, M. De Lisio, J. C. Chiao, S.-Jie Li and D. B. Rutledge are with the Department of Electrical Engineering, California Institute of Technology, Pasadena, CA 91125.

IEEE Log Number 9211932.

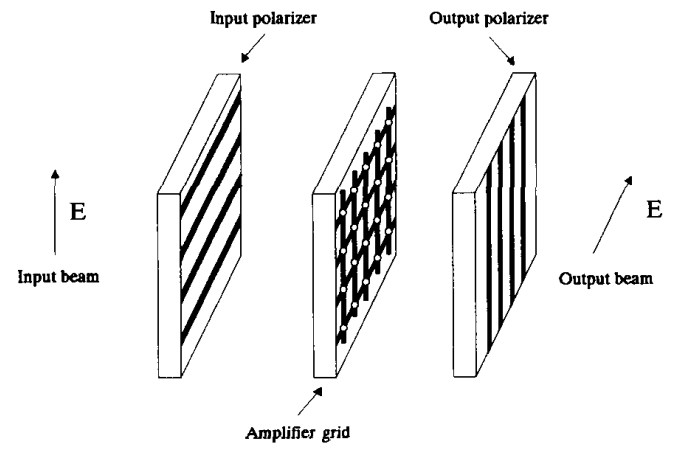

Fig. 1. A perspective view of a grid amplifier. The input beam is vertically polarized and the output beam is horizontally polarized. The metal strips on the grid couple the beam to the active devices, and radiate an amplified beam through the output polarizer. The position of the polarizers determines the input and output matching frequency.

necessary for bias, but they also caused the grid to have a 3 -dB bandwidth of only $3 \%$. This configuration was also not a good candidate for future monolithic integration. Furthermore, the transistors were packaged devices. This forces the unit cell to be large, restricting operation to a relatively low frequency. More recently, a 16-element planar grid with custom differential-pair heterojunction-bipolar-transistor (HBT) chips was demonstrated, with a gain of $11 \mathrm{~dB}$ at $10 \mathrm{GHz}$ [9]. However, this grid was small, little more than a wavelength across, and this made other measurements difficult. Here we report a much larger 100 -element amplifier that is nearly three wavelengths across. We have made measurements of gain, input and output match, saturated power, noise figure, modulation, failure tolerance, and angled beams.

\section{CONSTRUCTION}

The AlGaAs/GaAs HBT's were fabricated by Rockwell International. The epitaxial layers were grown by MOCVD. Table I gives the details. Carbon and silicon are the p-type and n-type dopants. The transistors were made by a self-aligned dual lift-off process [13]. The emitter area is $40 \mu \mathrm{m}^{2}$. The base resistance is estimated to be $10 \Omega$ and the base-collector capacitance to be $35 \mathrm{fF}$. A single HBT (Fig. 2(a)), biased at $I_{c}=20 \mathrm{~mA}$ and $V_{c e}=2.5 \mathrm{~V}$, had a dc common-emitter current gain, $\beta$, of 10 . The common-emitter power gain, $\left|s_{21}\right|^{2}$, was measured by Cascade probe, and was $11 \mathrm{~dB}$ at 
TABLE I

The Layers in the $\mathrm{Al}_{x} \mathrm{Ga}_{1-x}$ As/GaAs HBT's (TheSe were Grown by MOCVD)

\begin{tabular}{|c|c|c|c|c|}
\hline Layer & Thickness, $\mu \mathrm{m}$ & Material & Doping, $\mathrm{cm}^{-3}$ & Content, $x$ \\
\hline Cap & $0.15-0.25$ & $n^{+}$ & $4-5 \times 10^{18}$ & 0.0 \\
\hline Emitter & $0.07-0.12$ & $\mathrm{n}$ & $3-7 \times 10^{17}$ & 0.25 \\
\hline Base & $0.05-0.10$ & $\mathrm{p}^{+}$ & $3-6 \times 10^{19}$ & 0.0 \\
\hline Collector & $0.50-0.90$ & n & $3-8 \times 10^{16}$ & 0.0 \\
\hline Subcollector & $0.60-0.90$ & $\mathrm{n}^{+}$ & $4-5 \times 10^{18}$ & 0.0 \\
\hline
\end{tabular}

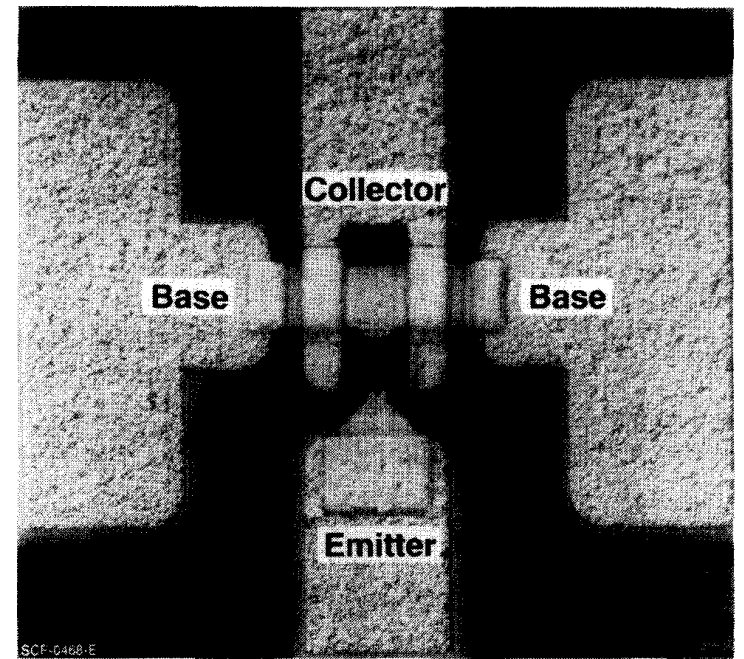

(a)

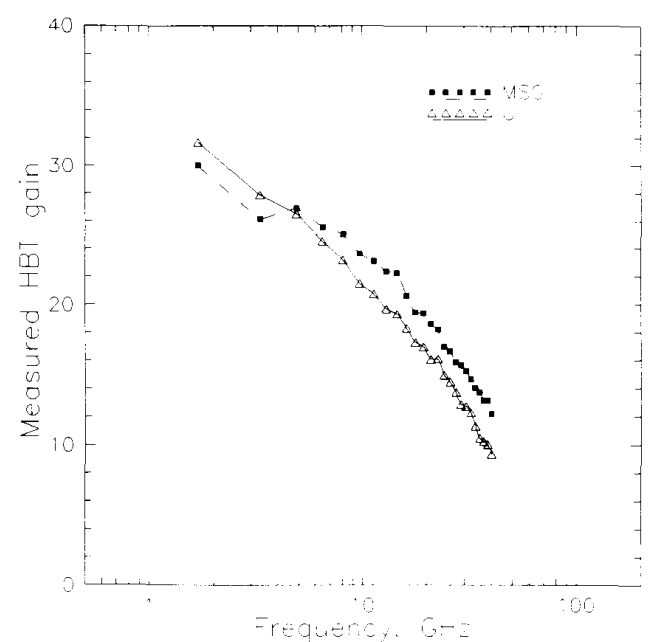

(b)

Fig. 2. (a) The layout of the single HBT used in device characterization measurements. There are two $10 \mu \mathrm{m} \times 2 \mu \mathrm{m}$ emitter fingers. (b) Maximum stable gain (MSG) and unilateral gain (U) versus frequency for this HBT.

$10 \mathrm{GHz}$, with a unilateral gain of $18 \mathrm{~dB}$ (Fig. 2(b)). The cutoff frequency, $f_{t}$, was $65 \mathrm{GHz}$, and the maximum frequency of oscillation, $f_{\max }$, was $90 \mathrm{GHz}$.

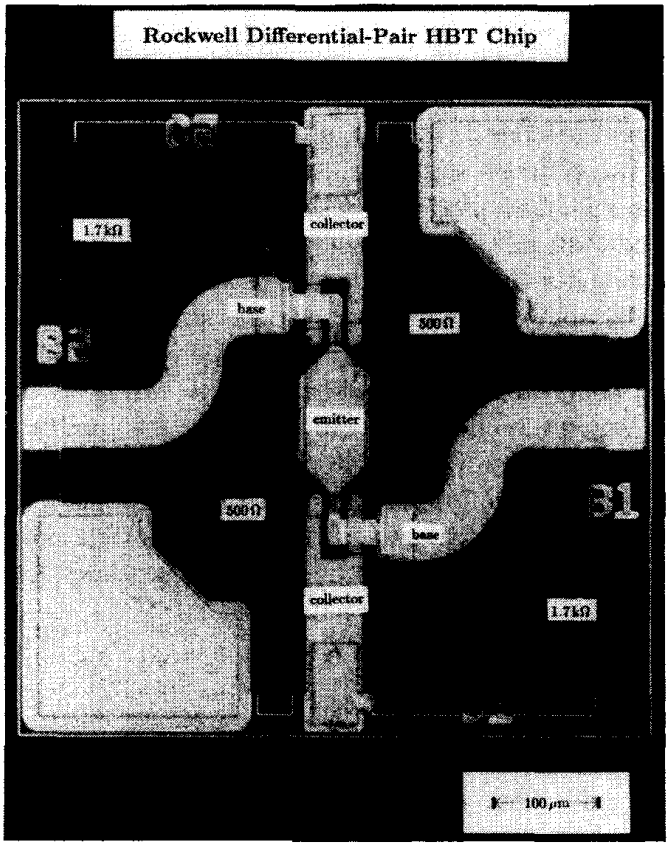

Fig. 3. The differential-pair HBT chip layout.

The differential-pair HBT chip used in the amplifier grid is shown in Fig. 3. The 1.7-k $\Omega$ resistors provide bias to the base, so there are only two external bias connections. They also provide negative feedback to help stabilize the grid. In addition, there are a pair of parallel $500-\Omega$ emitter resistors to reduce the common-mode gain to prevent the grid from oscillating. The values of the resistors were chosen to provide a bias of $V_{c e}=3 \mathrm{~V}$ and $I_{e}=11 \mathrm{~mA}$, assuming a $\beta$ of 10. During the grid measurements, a bias of $5 \mathrm{~V}$ and $860 \mathrm{~mA}$ was applied to the entire 100-chip grid. This means that the average emitter current was only $4.3 \mathrm{~mA}$, indicating that the chip $\beta$ was less than half that of a single HBT. We do not know why the $\beta$ 's are so different. All the devices came from the same wafer. The chips were passivated with silicon dioxide, but there was a drop in grid gain during the six-month period that the grids were being tested. This could be caused by device degradation or failure of the individual chips. In any event, we would expect the RF gain of the amplifier grid to be smaller than that of the single HBT chips. 
The metal structure in the grid has two parts: RF coupling for the input and output, and bias for the HBT chips. Our goal was to put both on the same surface. For input and output coupling, inductive strips are attractive because they are strongly polarized, providing good isolation between the input and the output to prevent unwanted oscillations. Ideally, the bias lines should have a large reactance so as not to disturb the input and output beams. Various metal patterns were tested on smaller $4 \times 4$ grids [14]. Best results were obtained from the unit-cell pattern shown in Figure 4(a). This design was then adopted for the $10 \times 10$ grid shown in Fig. 4(b). Inductive strips couple the chips to the input and output beams, and $0.1-\mathrm{mm}$ wide meander lines provide bias. A $0.1-\mathrm{mm}$ wide capacitive gap is etched in the input strips to tune out inductance. To evaluate the behavior of the meander lines, two different structures, each containing a narrow metal strip in a 2-mm square TEM waveguide were examined on the Hewlett-Packard High Frequency Structure Simulator (Fig. 5). The meander line had an inductive reactance of $100 \Omega$ at $10 \mathrm{GHz}$, compared with $50 \Omega$ for the straight strip. Similar ratios were obtained on different size waveguides [14].

The completed grid amplifier is shown in Fig. 6. Initially there was an oscillation at $10 \mathrm{GHz}$, but this problem was solved by adding a Duroid substrate with a dielectric constant of 10.5 behind the grid. There is also an additional dielectric slab at the output for tuning. The positions of the polarizers were carefully adjusted to produce the largest amplifier gain at $10 \mathrm{GHz}$.

\section{GAIN AND RETURN Loss}

The grid gain, and input and output return loss, were measured by a quasi-optical network analyzer with focusing lenses [15]. The measurement setup includes an HP8510B network analyzer, two feed horns, and two teflon lenses (Fig. 7). The lenses have a diameter of $30 \mathrm{~cm}$, and focus the beam at a point $46 \mathrm{~cm}$ from the lens. At $10 \mathrm{GHz}$ the beamwaist diameter is $10 \mathrm{~cm}$. In order to measure the amplifier gain, the system was first calibrated for transmission loss using an absorber screen with a square hole, $8 \mathrm{~cm}$ on a side to match the amplifier grid (Fig. 7(a)). This absorber intercepts about half of the power in the focused beam. After the calibration, the amplifier grid was inserted, and the receiving horn was rotated $90^{\circ}$ to match the amplified beam polarization (Fig. 7(b)). The absorber was not required around the amplifier grid because the transmitting horn and the receiving horn were crosspolarized. The results show that the peak gain was $10 \mathrm{~dB}$ at $10 \mathrm{GHz}$ with a $3-\mathrm{dB}$ bandwidth of $1 \mathrm{GHz}$ (Fig. 8). The peak could be shifted from $8 \mathrm{GHz}$ to $11 \mathrm{GHz}$ by adjusting the polarizers. With no bias, the gain dropped below $-8 \mathrm{~dB}$ everywhere.

A similar arrangement with one horn and one lens served as a reflectometer to measure input and output return loss. The calibration standards were an absorber load, a metal short, and a 1-cm offset short. The results in Fig. 9 indicate that both the input and the output return loss were better than $15 \mathrm{~dB}$ at $10 \mathrm{GHz}$. The output match is narrower than the input match.

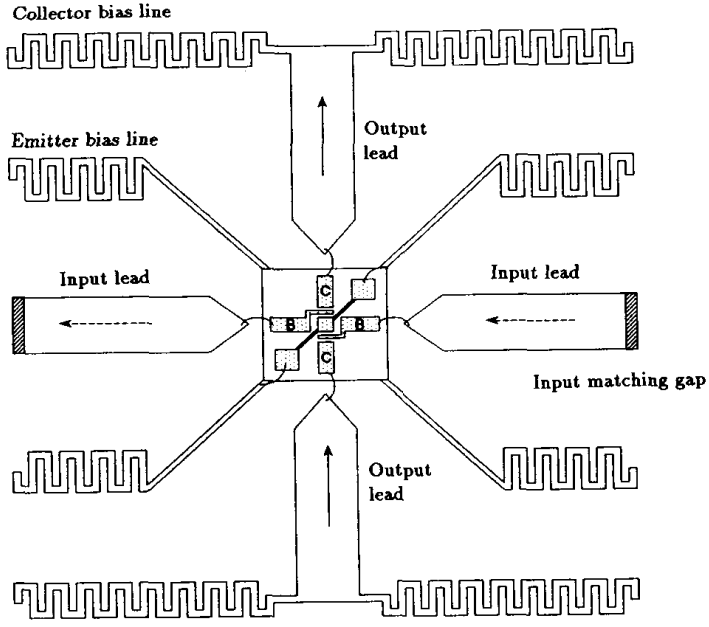

(a)

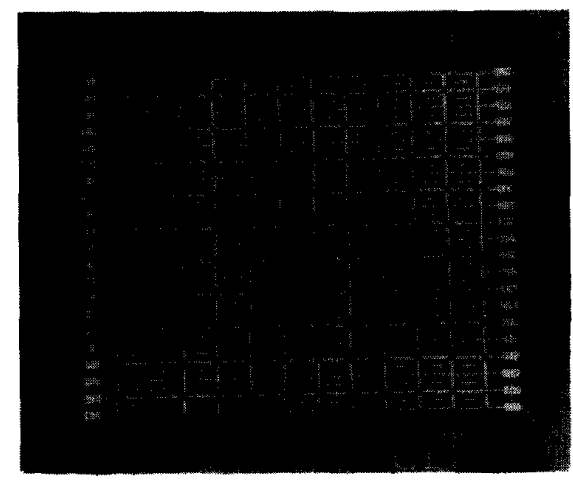

(b)

Fig. 4. (a) Grid-amplifier unit-cell design. The arrows indicate the direction of current flow. The unit cell size is $8 \mathrm{~mm}$. The radiating leads are $0.8 \mathrm{~mm}$ wide and the meandering bias lines are $0.1 \mathrm{~mm}$ wide. The span of the meander lines is $0.6 \mathrm{~mm}$ from top to bottom with $0.1-\mathrm{mm}$ gaps. The input matching gap is $0.1 \mathrm{~mm}$ wide. (b) Photograph of the 100-element grid amplifier.

Possibly this is because the output beam must pass through the high-dielectric constant Duroid slab under the grid.

One can also measure the amplifier gain in the far field without lenses with the setup in Fig. 10 [8]. The gain $G$ can be expressed in terms of the ratio of the calibration power, $P_{c}$, measured in (a), and the power received from the grid amplifier, $P_{r}$, measured in (b), as

$$
G=\frac{P_{r}}{P_{c}}\left(\frac{\lambda r}{2 A}\right)^{2}
$$

where $\lambda$ is the free-space wavelength and $r$ is the distance between the grid and each of the horns $(90 \mathrm{~cm})$. A is the geometric area of the grid, $64 \mathrm{~cm}^{2}$. The results were almost identical to the previous measurements using lenses, showing a peak gain of $10 \mathrm{~dB}$ at $10 \mathrm{GHz}$ with a 3-dB bandwidth of $1 \mathrm{GHz}$. 


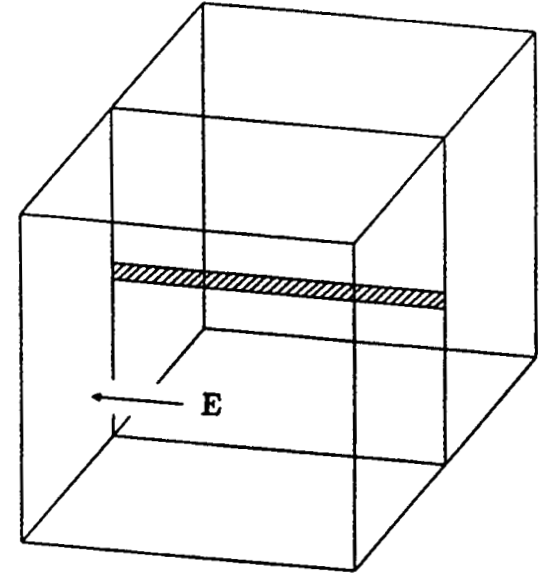

(a)

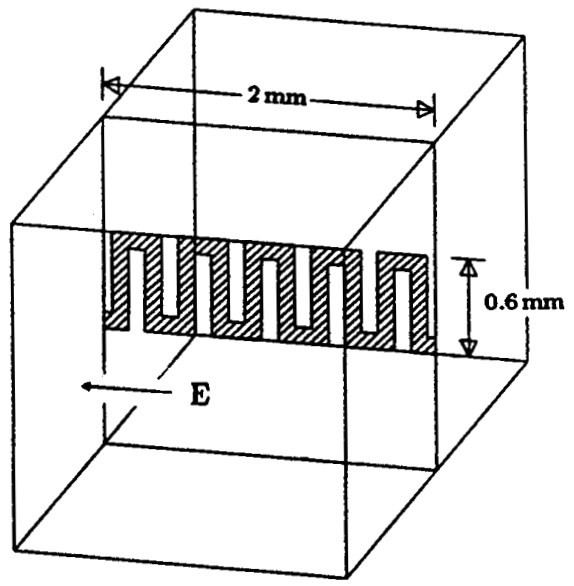

(b)

Fig. 5. High-Frequency Structure Simulator bias-line models in 2-mm TEM waveguide. (a) A straight strip. (b) A meander line. The sides of the waveguides are electric walls, and the top and bottom are magnetic walls, where the tangential component of the magnetic field goes to zero. The strips sit on a dielectric with $\epsilon_{r}=2.2$. The results show that between $7 \mathrm{GHz}$ and $13 \mathrm{GHz}$, the inductance of the meander lines is $1.6 \mathrm{nH}$, compared with only $0.8 \mathrm{nH}$ for the straight strip [14].

\section{POWER SATURATION}

In the far-field setup above, the incident beam has a uniform phase and amplitude, suitable for measuring power saturation. We need to know the gain of the transmitting and receiving horns, $G_{t}$ and $G_{r}$. These gains were assumed to be identical, and the setup in Fig. 10(a) was used to calculate the horn gain as

$$
G_{t}=G_{r}=\sqrt{\frac{P_{c}}{P_{t}}}\left(\frac{4 \pi 2 r}{\lambda}\right)
$$

where $P_{t}$ is the transmitted power. We can write the input power to the grid, $P_{i}$, as

$$
P_{i}=P_{t}\left(\frac{G_{t} A}{4 \pi r^{2}}\right)
$$

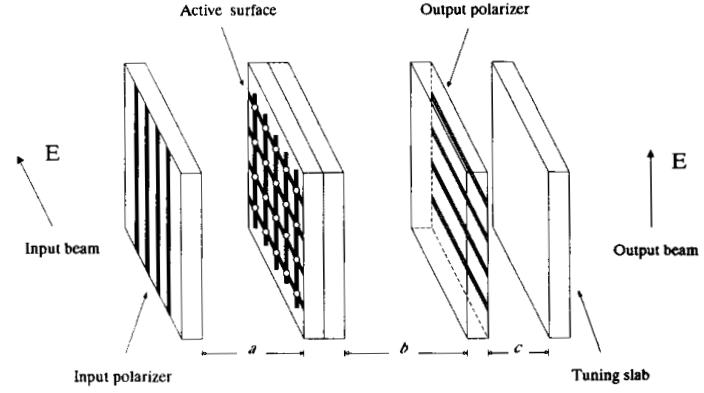

\begin{tabular}{|c|c|c|c|}
\hline & $\begin{array}{c}\text { dielectric } \\
\text { constant }\end{array}$ & $\begin{array}{c}\text { Physical } \\
\text { length, mm }\end{array}$ & $\begin{array}{c}\text { Electrical } \\
\text { length, 10 GHz }\end{array}$ \\
\hline$a$ & 1 & 14 & $168^{\circ}$ \\
\hline$b$ & 1 & 15 & $180^{\circ}$ \\
\hline$c$ & 1 & 10 & $120^{\circ}$ \\
\hline$\square$ & 10.5 & 3.81 & $148^{\circ}$ \\
\hline[] & 2.2 & 3.18 & $57^{\circ}$ \\
\hline
\end{tabular}

Fig. 6. View of the assembled grid amplifier. The table shows the slab thicknesses and optimum spacings for maximum gain at $10 \mathrm{GHz}$.

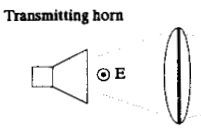

Transmitting horn
Ahsorber screen

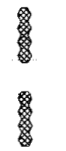

(a)

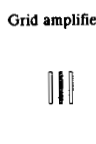

\|\|

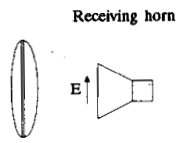

(b)

Fig. 7. Quasi-optical gain measurements with focusing lenses. (a) Transmission loss calibration. (b) Amplifier gain measurement.

We can write a related expression for the output power, $P_{o}$, as

$$
P_{o}=P_{r}\left(\frac{4 \pi r^{2}}{G_{r} A}\right)
$$

Fig. 11(a) shows the output power at $10 \mathrm{GHz}$, with the polarizers and slabs in the same positions as before. At a $5-\mathrm{V}$ bias, the $1-\mathrm{dB}$ compression point was $105 \mathrm{~mW}$ and the maximum output power was $450 \mathrm{~mW}$. The maximum poweradded-efficiency was $5 \%$, at an output power level of $330 \mathrm{~mW}$. The dc power supplied to the grid was $4.3 \mathrm{~W}$. We estimate that half the power was consumed in the base and emitter resistors, indicating that an active bias network that avoids this loss would help. The maximum output power decreased sharply as the frequency was changed from $10 \mathrm{GHz}$ (Figure 11(b)). The peak in output power comes at the same frequency where the output was matched. 


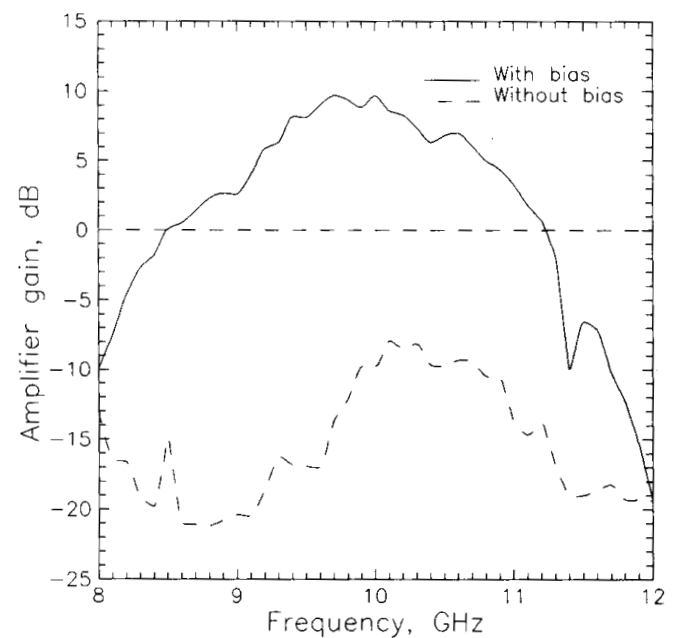

Fig. 8. Grid-amplifier gain versus frequency. The peak is $10 \mathrm{~dB}$ at $10 \mathrm{GHz}$. The $3-\mathrm{dB}$ gain bandwidth is $1 \mathrm{GHz}$.

\section{NOISE-FIGURE}

The far-field setup can also be used to measure the grid noise figure if the transmitting signal generator is replaced by a noise source, and the receiving power meter is replaced by a noise-figure meter. A noise diode with a high excess noise ratio (ENR) is required. We used a Noise-Com NC3206 diode, which has an ENR of $36 \mathrm{~dB}$. In the measurements, there is a noise contribution from background radiation, and a calibration measurement is made using the setup in Fig. 10(a) to correct for this. The antenna temperature at the receiving horn, $T_{c}$, can be expressed as a contribution from the noise source, with temperature $T_{t}$, and the effective background temperature $T_{b}$,

$$
T_{c}=L_{c} T_{t}+\left(1-L_{c}\right) T_{b}
$$

where $L_{c}$ is the space-loss factor $G_{t} G_{r}(\lambda / 4 \pi 2 r)^{2}$. The noise contributed by the horns themselves is neglected. From the meter measurements, we can invert this formula to find $T_{b}$. The results, shown in Fig. 12(a), indicate that the room background varied between $250 \mathrm{~K}$ and $380 \mathrm{~K}$. To measure the noise temperature of the grid, we use the arrangement in Fig. 10(b). The temperature of the receiving horn, $T_{r}$, is given in terms of $T_{t}, T_{b}$, and the noise temperature of the grid $T$ as

$$
T_{r}=G L T+G L^{2} T_{t}+(1-L)(G L+1) T_{b}
$$

where $G$ is the grid gain, and $L$ is the space-loss factor $G_{t} A /\left(4 \pi r^{2}\right)=G_{r} A /\left(4 \pi r^{2}\right)$. From the measurements, this formula can be inverted to find $T$. The results, converted to noise figure using a reference temperature $T_{0}=290 \mathrm{~K}$, are plotted in Fig. 12(b). The best noise figure at $5-\mathrm{V}$ bias was $7 \mathrm{~dB}$. When the bias was lowered to $4 \mathrm{~V}$, the noise figure improved to $6 \mathrm{~dB}$, but the gain also dropped by $1 \mathrm{~dB}$. For comparison, an optimized single HBT transistor circuit gave a noise figure of $4 \mathrm{~dB}$ [16]. In our measurements, the grid noise and the background noise were comparable, and we feel this limits the accuracy of the measurements to only $\pm 1 \mathrm{~dB}$. For future low-noise grids based on HEMT amplifiers, it would

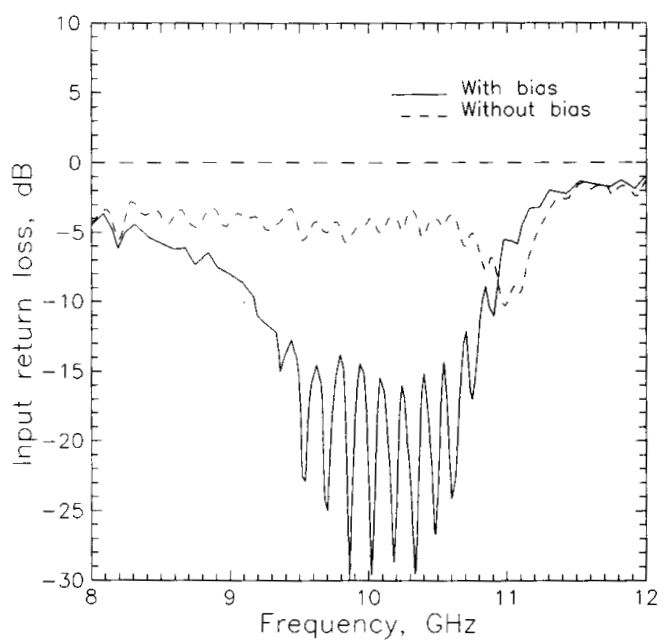

(a)

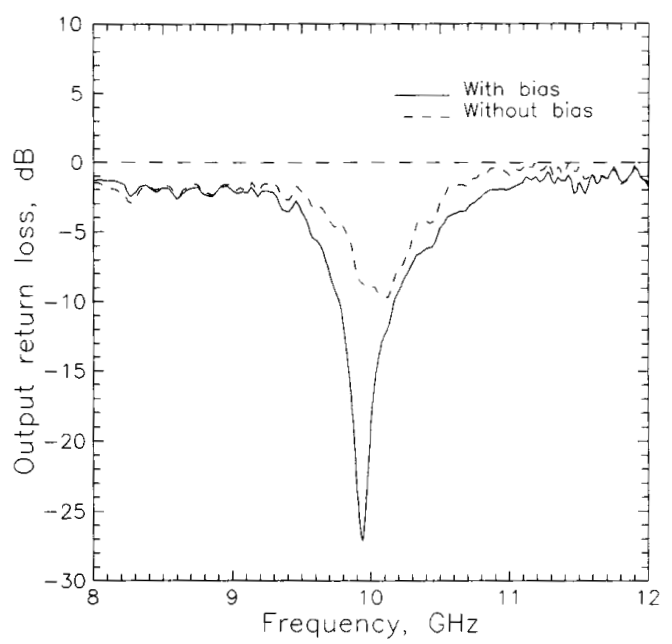

(b)

Fig. 9. (a) Input and (b) output return loss. The ripple in the input plot indicates that some reflected component was not accurately corrected for.

be better to measure noise figure with a cold background such as the sky.

\section{AMPLITUde Modulation}

The grid gain varies with bias, and this can be used for amplitude modulation. In Fig. 13(a), we plot the square root of the power gain $G$-the field transfer coefficient, $\left|s_{21}\right|-$-as a function of the bias voltage. Near $2 \mathrm{~V}$, we can write a linear approximation for this curve as

$$
\left|s_{21}\right|=1.16(1+0.82 v)
$$




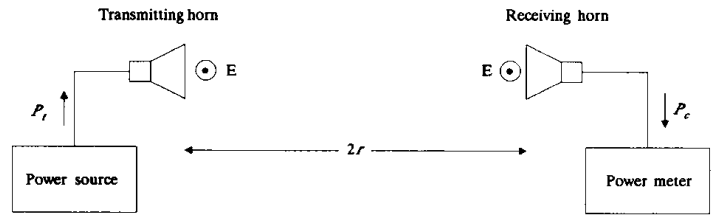

(a)

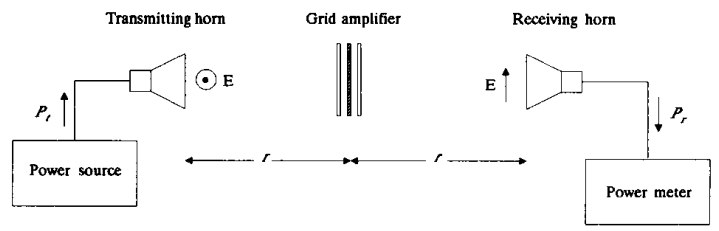

(b)

Fig. 10. Quasi-optical gain measurements in the far field. (a) Transmission loss calibration. (b) Amplifier gain measurement.

where $v$ is the voltage offset from $2 \mathrm{~V}$. We can write an amplitude-modulated signal $x(t)$ in the form

$$
x(t)=A\left[1+m \cos \left(\omega_{m} t\right)\right] \cos (\omega t)
$$

where $m$ is the modulation index and $\omega_{m}$ is the modulation frequency. If we compare these two equations, we can see that the modulation index $m$ should be given by $m=0.82 v$, if we consider $v$ to be the peak amplitude of the modulating signal. Fig. 13(b) compares measurements of $m$ with this prediction. At low modulation indexes, theory and experiment agree, but as $m$ approaches 0.5 , the points diverge. Also, additional sidebands begin to appear in the output spectrum [14].

\section{RELIABILITY}

A key issue in any system is how it responds to failure. Previously, a failure test was performed on a grid oscillator with 36 transistors by physically removing transistors from the grid [17]. The output power dropped quickly, by $2 \mathrm{~dB}$ per transistor. However, it is not clear how these results apply to grid amplifiers. Intuitively, we might expect each device to contribute equally to the radiated field, and the effect of a particular failure rate should show up as a corresponding drop in the radiated field. Thus a $10 \%$ failure rate would cause a $10 \%$ drop in the output field, and a $1-\mathrm{dB}$ drop in output power.

Bias is another reliability issue. If all the devices are biased in parallel, a short brings down the entire grid. In principle, this can be avoided by fusing individual bias lines. This problem did not occur during the six-month testing period. A number of devices failed open and the only effect was a small drop in gain. As a practical matter, bias failures in an HBT grid can be detected with an infrared viewer. When an HBT chip is properly biased, minority carriers recombine in the base, producing infrared radiation. This allows us to replace chips that are not biased, although it does not guarantee that all the chips are working.

To study failures, we detuned some of the base inputs by closing the input matching gaps with bond wires. These

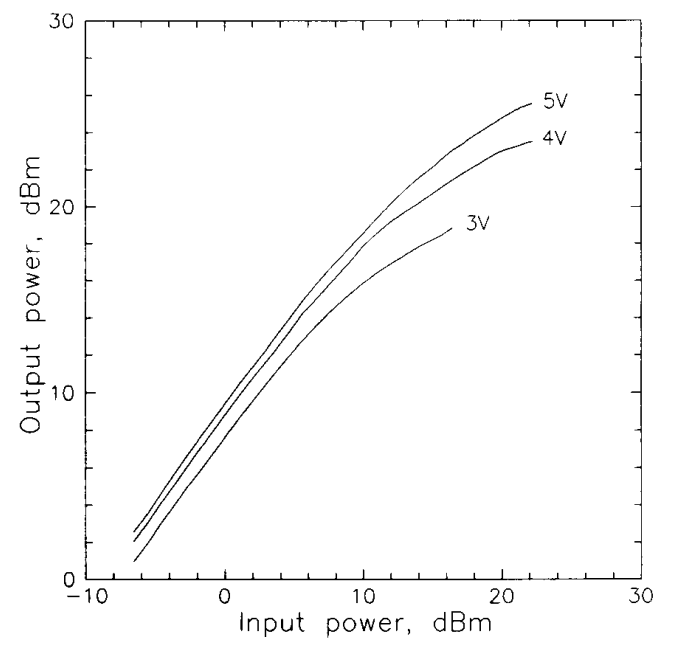

(a)

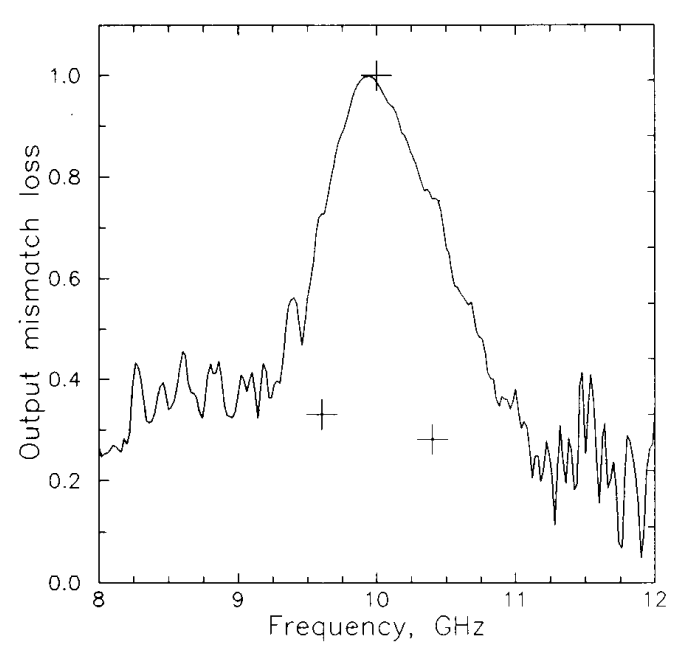

(b)

Fig. 11. Power saturation measurements. (a) Output power at $10 \mathrm{GHz}$ plotted against input power, for three different grid bias voltages. (b) Output mismatch loss factor, $1-\left|s_{22}\right|^{2}$, plotted against frequency. For comparison, the maximum output power, measured at three different frequencies, is also plotted, normalized to the same scale. The grid bias voltage in each case was $5 \mathrm{~V}$.

input matching gaps are $0.1 \mathrm{~mm}$ wide, and provide series capacitance for tuning out the inductance of the base lead (see Fig. 4(a)). Each bond wire detunes 2 of the 200 base circuits, so that we can produce a $10 \%$ failure rate by adding 10 bond wires. The bond wires were placed uniformly across the grid. The bonds did not result in any spurious oscillations. Fig. 14 shows the effect on the gain. At the normal bias voltage of $5 \mathrm{~V}$, the gain dropped $1 \mathrm{~dB}$ with a $10 \%$ failure rate. With a $25 \%$ failure rate, the drop was $5 \mathrm{~dB}$, and at $50 \%$, the gain fell all the way to the normal zero-bias gain. 


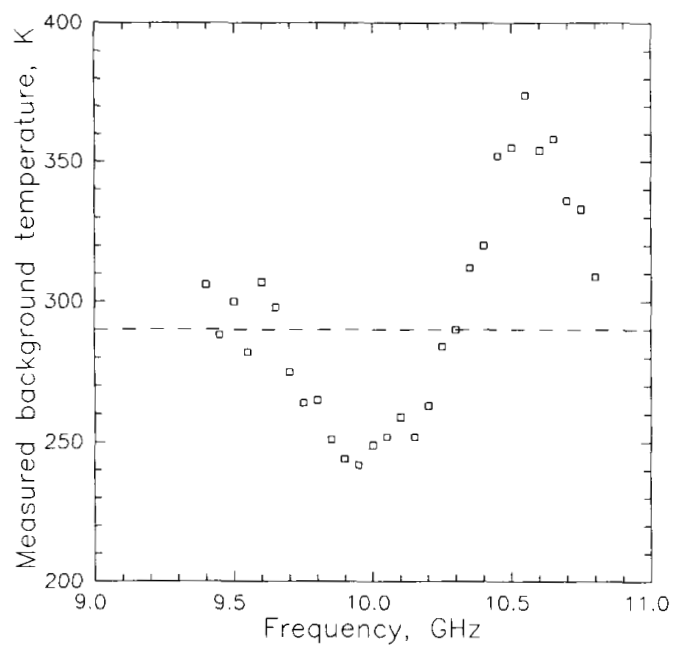

(a)

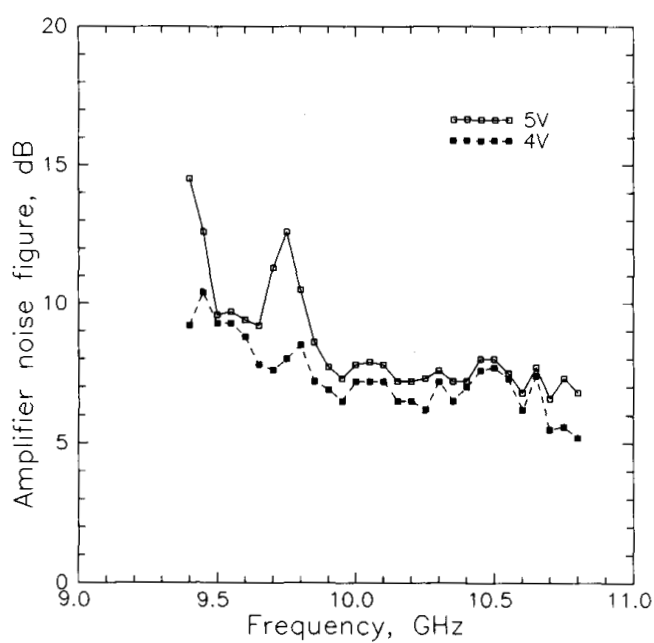

(b)

Fig. 12. (a) Effective background temperature $T_{b}$. (b) Grid noise figure at two different bias voltages.

\section{BEAM PATTERNS}

The grid amplifier is nearly three wavelengths across, and we would expect that if the phase and amplitude of the input beam vary across the grid, then the phase and amplitude of the output beam would vary in the same way. Diverging input beams should produce diverging output beams and the angle of a beam should be preserved as it is amplified. Practically, this means that beams can be formed in a transmitter at a lowpower level with a particular direction, width and sidelobe level, and then amplified.

To test this idea we used a small 8-transistor HEMT grid oscillator as a source. Its H-plane pattern is shown in Fig. 15(a) as a solid line. The oscillator was then moved back $5 \mathrm{~cm}$ $(1.6 \lambda)$ to allow the grid amplifier to be inserted. The pattern of the amplified beam (this is the E-plane pattern because

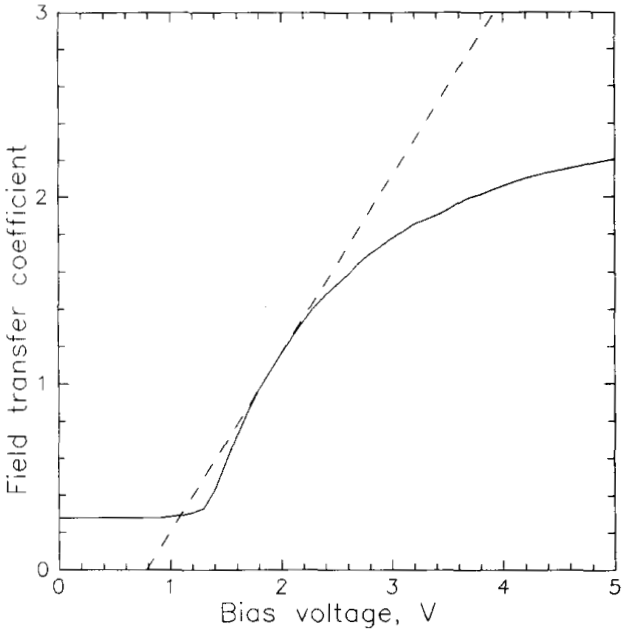

(a)

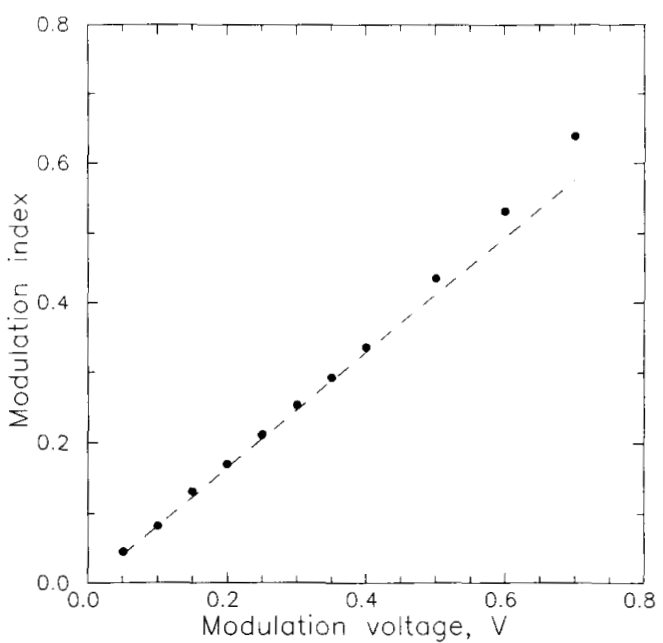

(b)

Fig. 13. Amplitude modulation by changing the grid bias. (a) The field transfer coefficient $\left|s_{21}\right|$, plotted on a linear scale. The dashed line is a linear approximation of the curve near $2 \mathrm{~V}$, given by $\left|s_{21}\right|=1.16(1+0.82 v)$. This measurement was made several months after the first measurements, and the gain has dropped. (b) Modulation index $m$ as a function of the peak amplitude $v$ of the modulating signal. The bias was $2 \mathrm{~V}$. The circles are the measured values, and the dashed line is the prediction $m=0.82 v$.

the grid changes the polarization) is shown as a dashed line in Fig. 15(a). Both patterns have relatively wide beams, with the amplified beam being somewhat narrower. The amplifier increased the peak power density by $15 \mathrm{~dB}$. Next the oscillator grid was moved back until the two grids were separated by $34 \mathrm{~cm}(11.4 \lambda)$. This amplified beam is the solid line in Fig. 15(b). The beam is narrower, because the input phase and amplitude are now nearly uniform. In this case the beamwidth is diffraction limited by the grid amplifier. This idea is supported by close agreement with a theoretical pattern calculated for a uniform array of 10 elementary dipoles $8 \mathrm{~mm}$ apart (the dashed line in Fig. 15(b)). 


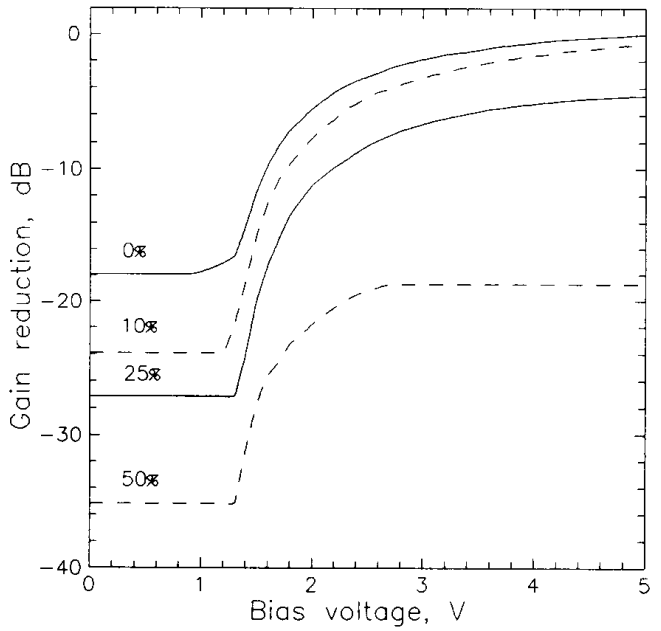

Fig. 14. Closing the input matching gaps with bond wires. The plot shows the measured gain at $10 \mathrm{GHz}$ as a function of the bias voltage, normalized to the gain at $5-\mathrm{V}$ bias. A $10 \%$ failure rate results in a gain drop of $1 \mathrm{~dB}$.

We also tested the amplifier with beams incident at different angles. Fig. 16(a) shows the patterns for incidence at $0^{\circ},+20^{\circ}$, and $-20^{\circ}$. The peaks in the output beams are within $1^{\circ}$ of the incident angles. The beams are all of similar width, and the sidelobes are all at about the $13-\mathrm{dB}$ level that we would expect for a uniformly illuminated aperture. In another test, we started with the far-field arrangement in Fig. 10(b), and rotated the grid amplifier. This measures the change in gain for different angles of incidence. The gain drops less than $3 \mathrm{~dB}$ for angles of incidence less than $30^{\circ}$. Presumably the primary cause of this drop in gain at extreme angles is changing beam impedance. However, part of the loss is due to the fact that less power is being intercepted by the grid at oblique angles. This obliquity factor shows up in both transmitting and receiving space-loss factors, so we expect a $\cos ^{2} \theta$ loss $\left(1.2 \mathrm{~dB}\right.$ at $\left.30^{\circ}\right)$ in addition to the drop caused by changing impedances. We can avoid this obliquity loss in applications where grid can be made larger than the beam.

\section{CONCLUSION}

We have presented a 100 -element HBT grid amplifier with a gain of $10 \mathrm{~dB}$ at $10 \mathrm{GHz}$, a $10 \% 3-\mathrm{dB}$ bandwidth, and an input and output match better than $15 \mathrm{~dB}$. At a $5-\mathrm{V}$ bias, the maximum output power is $450 \mathrm{~mW}$ and the minimum noise figure is $7 \mathrm{~dB}$. These results are consistent with the idea that the gain, bandwidth, input and output match, and noise figure are comparable to single-transistor amplifiers, but that power scales with the size of the grid. The grid degrades gracefully $-10 \%$ failures produce only a $1-\mathrm{dB}$ drop in gain. This means that grid amplifier could be much more reliable than a single high-power solid-state device or vacuum tube. A grid amplifier is a multi-mode device that preserves the propagation angles of beams. It amplifies at incident angles

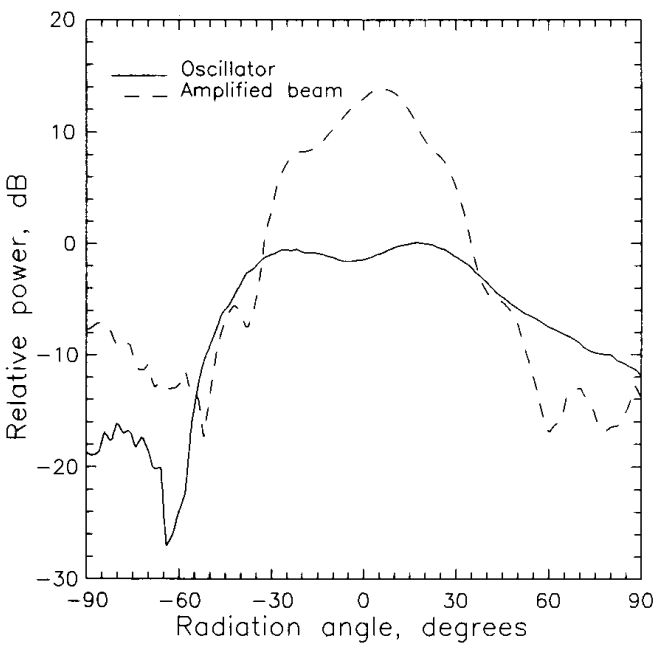

(a)

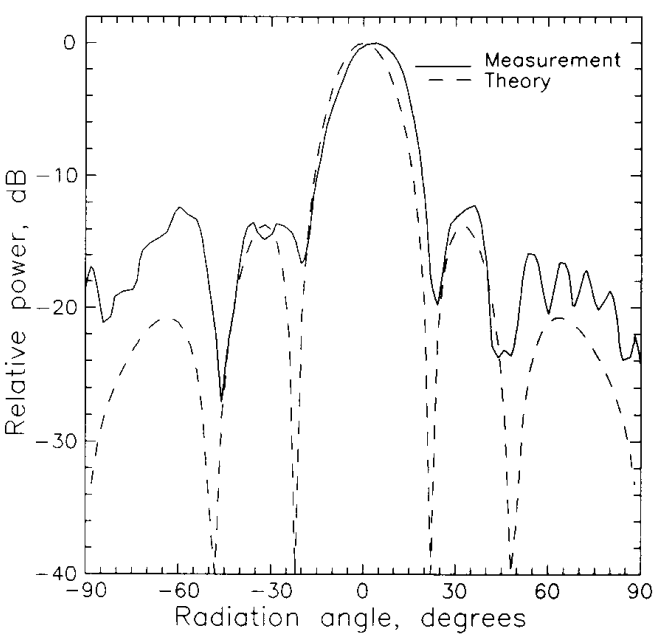

(b)

Fig. 15. Amplifying the beam of a small $10-\mathrm{GHz}$ grid oscillator with a grid amplifier. (a) H-plane pattern for the oscillator grid (solid line), and the E-plane pattern for the amplified beam (dashed line). The distance between the two grids was $1.6 \lambda$. Notice that both the grid-oscillator beam and the grid-amplifier beam are broad. (b) Spacing increased to $11.4 \lambda$. The solid line is the E-plane pattern of the amplified beam, now narrowed because the input phase and amplitude are uniform. The dashed line shows the theoretical pattern of a uniform array of 10 elementary dipoles $8 \mathrm{~mm}$ apart.

up to $30^{\circ}$ with less than a $3-\mathrm{dB}$ loss in gain. This means that a grid amplifier could amplify a beam for an electronic beam steerer, easing the loss requirements for the steering system. Recently a monolithic grid oscillator has been demonstrated at $35 \mathrm{GHz}$ [18]. This grid-amplifier design is also suitable for monolithic integration at millimeter wavelengths. 


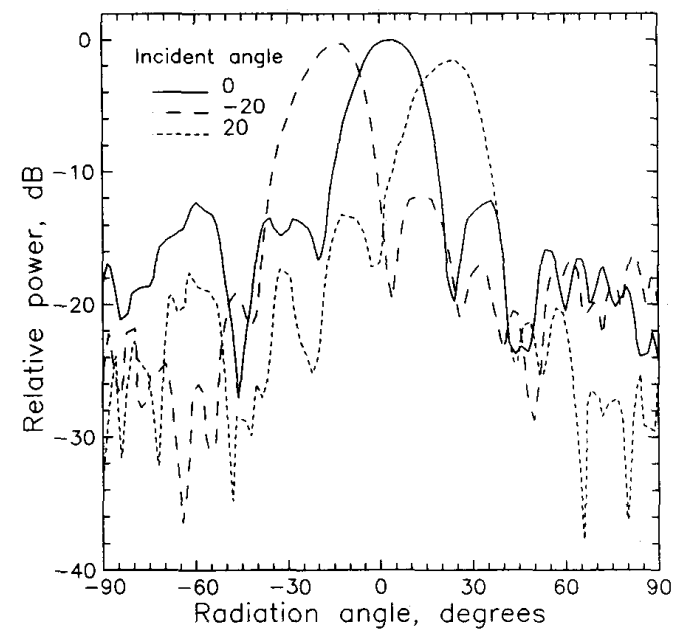

(a)

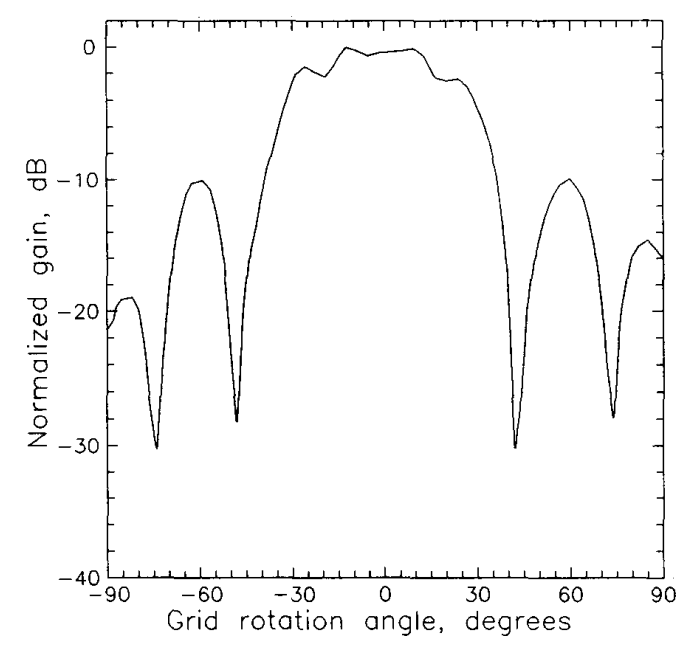

(b)

Fig. 16. Amplifying beams at angles. (a) The grid-amplifier radiation patterns for three different incident beams (b) Gain variation with incidence angle.
[6] Z.B. Popović, R. M. Weikle, II, M. Kim, and D. B. Rutledge, "A 100MESFET planar grid oscillator," IEEE Trans. Microwave Theory Tech., vol. 39, pp. 193-200, 1991.

[7] R.M. Weikle, II, M. Kim, J. B. Hacker, M.P. De Lisio, and D. B. Rutledge, "Planar MESFET grid oscillators using gate feedback," IEEE Trans. Microwave Theory Tech, vol. 40, pp. 1997-2003, 1992.

[8] M. Kim, J. J. Rosenberg, R. P. Smith, R. M. Weikle, II, J. B. Hacker, M. P. De Lisio, and D. B. Rutledge, "A grid amplifier," IEEE Microwave Guided Wave Lett., vol. 1, pp. 322-324, 1991.

[9] D. B. Rutledge, J. B. Hacker, M. Kim, R. M. Weikle, II, R. P. Smith, E. A. Sovero, "Oscillator and amplifier grids," IEEE MTT-S Int. Microwave Symp. Dig. Albuquerque, NM, 1992, pp. 815-817.

[10] J.B. Hacker, R.M. Weikle, II, M. Kim, M.P. De Lisio, and D.B. Rutledge, "A 100-element Schottky diode-grid mixer," IEEE Trans. Microwave Theory Tech., vol. 40, pp. 557-562, 1992.

[11] K. Stephan, F. Spooner, and P. Goldsmith, "Quasi-optical millimeter wave hybrid and monolithic PIN diode switches," submitted to IEEE Trans. Microwave Theory Tech.

[12] L. B. Sjogren, H. -X.L. Liu, X.H. Quin, W. H. Wu, E. Chung, C Domier, and N.C. Luhmann, Jr., "A monolithic millimeter-wave diode array beam transmittance controller," submitted to IEEE Trans. Microwave Theory Tech.

[13] M. Chang et al., "AlGaAs/GaAs heterojunction bipolar transistors fabricated using a self-aligned dual-lift-off process," IEEE Electron Device Lett. vol. EDL-8, pp. 303-305, 1987.

[14] M. Kim, "Grid amplifiers," Ph.D. thesis, California Institute of Technology, Pasadena CA, 1992.

[15] D. Gagnon, "Highly sensitive measurements with a lens focused reflectometer," IEEE Trans. Microwave Theory Tech., vol. 39, pp. 22372240, 1991.

[16] P. M. Asbeck, M. Chung, F. Chang, J. A. Higgins, N.H. Sheng, G. J. Sullivan, and K. C. Wang, "GaAsAl/GaAs heterojunction bipolar transistors: issues and prospects for application," IEEE Trans. Electron Devices, vol. 36, pp. 2032-2035, 1989.

[17] Z. B. Popović, R.M. Weikle, II, M. Kim, K. A. Potter, and D. B. Rutledge, "Bar-grid oscillators," IEEE Trans. Microwave Theory Tech., vol. 38, pp. 225-230, 1990.

[18] M. Kim, E. A. Sovero, R. M. Weikle, J. B. Hacker, M. P. De Lisio, and D. B. Rutledge, "A 35-GHz HBT monolithic grid oscillator," in 17th Int. Conf. on Infrared and Millimeter Waves, R. J. Temkin, Ed., Proc. SPIE 1929, pp. 402-403, 1992.

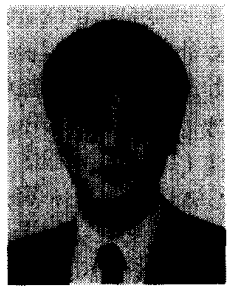

Moonil Kim (S'91-M'93) was born in Seoul, Korea on March 14, 1965. He received the B.S degree from the Illinois Institute of Technology in 1987, and the M.S. and Ph.D. degrees from the California Institute of Technology in 1988 and 1993, respectively.

Currently, he is with the Central Research Laboratory, Gold Star in Seoul, Korea. His main research interest is building and testing quasioptical millimeter-wave components using solid state devices.

\section{REFERENCES}

[1] D. B. Rutledge and S. E. Schwarz, "Multimode antennas for far-infrared and millimeter-wave detectors," IEEE J. Quantum Electron. vol. QE-17, pp. 407-414, 1981.

[2] W. W. Lam, C.F. Jou, H. Z. Chen, K.S. Stolt, N. C. Luhmann, Jr., and D. B. Rutledge, "Millimeter-wave diode-grid phase shifters," IEEE Trans. Microwave Theory Tech. vol. 36, pp. 902-907, 1988.

[3] Lance Sjogren, "A multi-function millimeter-wave Schottky-diode array electronic beam controller," Ph.D. thesis, UCLA, Los Angeles CA, 1993.

[4] C. F. Jou, W. W. Lam, H. Z. Chen, K. S. Stolt, N. C. Luhmann, Jr., and D. B. Rutledge, "Millimeter-wave diode-grid frequency doubler," IEEE Trans. Microwave Theory Tech. vol. 36, pp. 1507-1514, 1988.

[5] E. I. Chung, H. -X. L. Liu, C. W. Domier, and N. C. Luhmann, Jr., "High power millimeter-wave quasi-optical frequency tripler arrays using resonant tunneling devices," in 17th Int. Conf. Infrared and Millimeter Waves, Pasadena CA, pp. 70-71, 1992. 


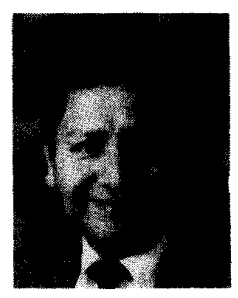

E. Sovero is a member of Technical Staff, Heterostructure FET Technology Department, Rockwell International Science Center. He holds a B.S., engineering, M.S., mechanical engineering, and Ph.D. applied physics and information science, California Institute of Technology, 1977.

Dr. Sovero joined Rockwell in 1977 . He initially worked on tunable acousto-optic and electro-optic spectral filters. These filters were used for image and signal processing with emphasis in the mid infrared. Since 1981, Dr. Sovero has been involved in the development of III-V devices (GaAs). His areas of particular expertise include the design, analysis and testing of active devices. His experience includes the experimentation demonstration of charge-coupled devices (CCD) used as building blocks of complex analog and optical signal processing functions. In recent years, Dr. Sovero has been involved in the development of HEMT and HBT millimeter-wavelength integrated circuits and devices, with particular emphasis on monolithic Quasi-Optic devices.

Dr. Sovero has authored or co-authored over 25 publications in the field of GaAs. He holds one patent in the area of electro-optical signal processing.

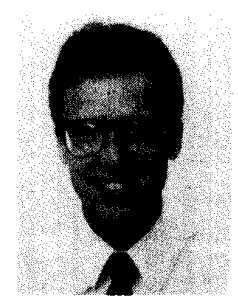

Jonathan B. Hacker (S'84) was born in Vancouver, British Columbia, Canada on February 16, 1963. He received the B.A.Sc. degree in electrical engineering in 1986 from the University of British Columbia, and the M.S. degree in electrical engineering from the California Institute of Technology, Pasadena in 1990. He is currently working towards the Ph.D. de gree in electrical engineering at Caltech. From 1986 to 1988 he was associated with the communication research and development group of the Nexus Engineering Corporation. His research interests include millimeter-wave quasi-optical techniques, microwave power amplifiers, and computer-aided design and measurement of microwave circuits

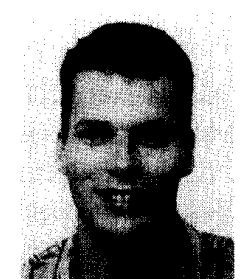

Michael P. De Lisio ( $\mathrm{S}^{\prime} 90-\mathrm{M}^{\prime} 92$ ) was born in Southfield, Michigan, on July 29, 1968. He received the B.S.E. degree in electrical engineering from the University of Michigan, Ann Arbor, in 1990. In 1991, he obtained the M.S. degree in electrical engineering from the California Institute of Tech nology in Pasadena. He is currently a candidate for the Ph.D. degree at Caltech. His research interests include high-frequency solid-state devices microwave and millimeter-wave power combining, and monolithic quasi-optical devices.

Mr. De Lisio is a National Science Foundation Graduate Research Fellow and is a member of Tau Beta Pi and Eta Kappa Nu.

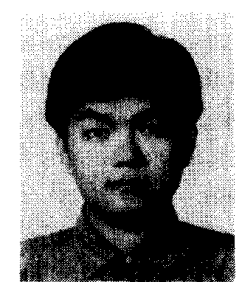

Jung-Chih Chiao was born in Tainan, Taiwan, Republic of China, in 1966. He received the B.S degree in electrical engineering from the National Taiwan University, Taipei, in 1988. From 1988 to 1990 , he served as a Technical Officer of Communication in the Air Force, Taipei. In 1991, he obtained the M.S. degree from the California Institute of Technology.

His research interests include monolithic millimeter-wave integrated-circuit antennas, submillimeter-wave frequency multipliers, and applications of micromachining techniques to beam-steering device. He is currently pursuing the Ph.D. degree at the California Institute of Technology.

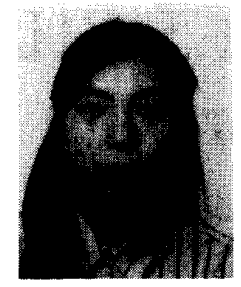

Shijie Lie was born in Beijing, P.R.China, on February 22, 1966. She received the B.S. and M.S. degrees in electrical engineering from Tsinghua University, Beijing, P.R.China, in 1988 and 1990 respectively. She is currently a candidate for the Ph.D. degree at Caltech. Her research interests include microwave and millimeter-wave power combining and beam-steering.

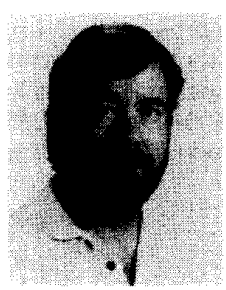

David R. Gagnon was born in Sacramento, CA on July 22,1951 . He received the M.S. degree in physics from Utah State University in 1984 and the M.S.E.E. (applied physics) degree from the University of California at San Diego in 1988. Since 1983, he has been a research physicist with the RF and Microwave Technology Branch of the Research Department at Naval Air Warfare Center, China Lake, CA. His work is primarily in the field of microwave measurement techniques. Mr. Gagnon is of Physics Teachers.

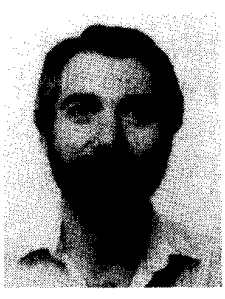

James J. Rosenberg was born in Paterson, New Jersey in 1957. He received the Sc.B. degree in engineering from Brown University in 1979, the M.S. degree in electrical engineering from the University of California, Berkeley, in 1980, and the Ph.D. degree in electrical engineering from Columbia University in 1983.

$\mathrm{He}$ has taught at Brown University and has worked at the Jet Propulsion Laboratory and at Germanium Power Devices Corporation. In 1992, he joined the faculty at Harvey Mudd College. His interests include high speed device design and fabrication, germanium device technology, and quasi-optical microwave amplifiers and oscillators.
David B. Rutledge, (M'75-SM'89-F'93) for a photograph and biography, see this issue, pp. 1662 